\title{
Implications of the Cellular Transport Step for Amino Acid Metabolism
}

\author{
by Halvor N. Christensen, Ph.D.
}

11 e do well to place the physicochemical step of amino acid transport on our metabolic diagrams, wherever it intervenes, on a par with the more purely chemical steps we assemble on such charts. This recommendation does not mean, as some suppose, that we merely insert the step of intestinal absorption between digestion and intermediary metabolism; it means that in addition we insert the step of the uptake and the modulated release of amino acids by the hepatocyte, along with the hepatic interconversions of amino acids and other hepatic contributions to their metabolism. It means we should consider the step of uptake and release by muscle along with muscle metabolism, the transfer across the bloodbrain barrier with brain metabolism, and the step of transfer into the renal epithelial cell from the blood plasma with amino acid uptake from the proximal tubular lumen and with the release phases of the same two transports. We are at a stage of the study of amino acid metabolism where we deal not merely with the aggregate effects of the four possibilities (synthesis, access, interconversion and degradation of amino acids) but we also need to consider the relative fluxes of all such events. Thus, we become able to recognize how dominant are some of the possible fluxes (e.g., of alanine from muscle to liver in gluconeogenesis ${ }^{1}$ ) and how small are some others. Also, we are able to know the steady state concentrations

Dr. Christensen is Professor, Department of Biochemistry, University of Michigan, Medical School, Ann Arbor, Michigan 48109.

A limited number of reprints of this article may be obtained from the author. THERE ARE NO REPRINTS OF UNSIGNED REVIEWS. maintained during these fluxes in the intervening extracellular fluid.

\section{Putting into Perspective Amino Acid Levels of the Blood Plasma}

Plasma analysis measures only a minor fraction of the amount of each amino acid present in the free state in the body. Besides, the fraction present in the plasma varies widely among the amino acids. For example, the proportion of glycine in the extracellular compartment is much smaller than that of leucine. Furthermore, the relation of the plasma concentration to the cellular concentration (for example in the liver) fluctuates considerably from hour to hour, and is highly subject to change with physiologic and nutritional conditions. We should also stress that the more decisive events of amino acid metabolism occur in the cells. Because of the convenience with which the blood plasma can be sampled, there is a great temptation to over-interpret the meaning of plasma analyses, and to attempt to develop uncertain generalizations from such values.

\section{Understanding Amino Acid Exodus from Cells}

Although the concept of the cellular uptake process seems to have been well assimilated into metabolic thinking, a difficulty may be noted in the comprehension of the release of amino acids from cells as a determined event, and indeed as part of the total event we call transport. The plasma membrane has rather little permeability to amino acids, whether inwards or outwards, that cannot be explained as specifically mediated. There is often a tendency to think that amino acid exodus at the steady 
state represents leakage, a simple diffusion just fast enough to compensate for the active transport inward. On the contrary, this exodus is mainly structurally specific and indeed may sometimes prove more stereospecific than entry. ${ }^{2,3}$ It can be shown to be largely saturable, and by inhibition analysis to occur by the same systems that produce entry, although clearly not in the same proportions among these systems. It is often not understood that when a transport system is operating as steeply uphill as the quantity of energy available to it allows, that system must, by the laws of thermodynamics, operate to let as many molecules out as in. The factor that usually prevents the more uphill systems from attaining the highest gradient of which they are capable at the real concentrations of the substances involved is not simple leakiness of the membrane, but the reversed contribution of a less strongly uphill system that handles the same solute in parallel to the one being examined. It can be shown that the gradient maintained by two such systems lies intermediate between the gradients each can maintain operating totally alone, ${ }^{4}$ rather than being greater than either of these two. Under these conditions the more uphill system produces net uptake, and the second allows net exodus, probably conserving at the same time some of the energy available from its reverse operation.

This situation seems quite useful and necessary for the epithelial cell of the intestine or kidney, where cells transfer amino acids and other substances across themselves, from the lumenal to the contralateral pole..$^{5}$ If the cell brush border is rich in units of the more uphill transport system, and the contralateral pole of the cell in the less uphill system, then the former acts to concentrate amino acids into the cell and the latter to release them again, but against a resistance such that the amino acid nutrition of the cell itself is not threatened.

A weakly uphill transport system may then be thought of as a means of restraining, through its reverse operation, the exodus of a nutrient. It is entirely likely at the same time that some of the energy invested in accumulating the nutrient within the cell is recovered by this reverse operation of an uphill transport. For cells not in an epithelium, the two transport systems under comparison presumably are randomly distributed over the cellular surface. That arrangement does not produce an orderly transcellular pumping, but it protects the nutrition of the cell and at the same time probably allows superior regulation of the degree of nutrient accumulation. The arrangement presents a generalized form of that proposed by Mitchell ${ }^{6}$ for the inner mitochondrial membrane, whereby it is believed that energetic coupling between electron transport and ATP synthesis can be obtained through the steady state operation of two $\mathrm{H}^{+}$-pumping systems; one runs forward and the other backward. In that case these pumping systems are, respectively, electron transport and the ATPase action corresponding to the reversal of oxidative phosphorylation.

I recently pointed out in an editorial letter in Nutrition Reviews ${ }^{7}$ the importance that must inevitably fall on the levels at which the various amino acids are held in each cell by this system of pumps. These concentrations are a priori just as important for the dispensable as for the indispensable amino acids in determining cellular events, for example in determining whether metabolic reactions take place. Furthermore, this importance of the steady-state levels maintained does not depend on whether an amino acid originates in the cell in question or from outside it. ${ }^{7}$

The role of the liver in controlling amino acid metabolism depends not simply on its uptake of amino acids but quite as much on its release of them. Regulation necessarily concerns one of these as much as the other. During the phase of hepatic release of amino acids, high concentration gradients are still maintained in favor of the interior. The principle follows: One cannot judge the net direction of a transport by the direction in which its gradient is oriented.

One interesting transport event actually has no physiologic importance, namely the exchange of one molecule of a solute across the membrane for another identical molecule of the same substance, apparently at no energy cost. But this aspect of transport reveals its importance when, for example, a leucine molecule exchanges for a phenylalanine molecule by way of the transport system. The regulation by the membrane and the hormones impinging on it of the direction and rate of net 
transport are the most interesting of all. For the liver the circadial rhythm of these changes represents a central nutritional and metabolic problem, one which has been discussed here previously. ${ }^{8}$ An old observation of such regula- amino acids show appreciable transport by both systems. Amino acids with short, polar or linear sidechains tend to be preferred by $\mathrm{Na}^{+}$-dependent System $A$, and those with branches or rings on the sidechain by $\mathrm{Na}^{+}$-

TABLE I

An Approximate Survey of $\alpha$-Amino

Acids with Several Transport Systems'

\begin{tabular}{lcccc}
\hline & \multicolumn{4}{c}{ System } \\
\cline { 2 - 4 } \multicolumn{1}{c}{ Amino acid } & Gly & A & ASC & $L$ \\
& & & & \\
\cline { 2 - 4 } Gly & +++ & ++ & \pm & + \\
Pro & In some cases & +++ & \pm & 0 \\
Ala, Ser & 0 & +++ & +++ & + \\
Thr, Homoser & 0 & +++ & ++++ & ++ \\
$\alpha$-Aminobutyric & 0 & ++ & +++ & ++ \\
GluNH & 0 & ++ & ++ & ++ \\
Norval & 0 & +++ & + & +++ \\
Norleu, Met & 0 & ++++ & 0 & ++++ \\
His, Tyr, Trp & 0 & ++ & 0 & ++ \\
Val & 0 & + & 0 & ++ \\
Leu, lle, Phe & 0 & + & 0 & ++++ \\
& & & + & ++ \\
\hline
\end{tabular}

'Reactivities are roughly compared as observed in the Ehrlich ascites tumor cell and in various red blood cells-see comment in text on differences to be expected in other tissues. A zero implies that little or no reactivity was observed under the ordinary conditions of comparison; under selected conditions significant reactivity may yet be observable. (11)

tion still has great interest: When a major portion of the rat liver is removed, the remaining portions intensify their accumulation of amino acids at just about the time when "regeneration" begins. ${ }^{9}$

\section{Systematization of Amino Acid Transport}

The above discussion assumes some knowledge of the various systems for the transport of amino acids in the cells of the higher animal. Four important transport systems for the neutral amino acids have been tabulated (Table I). Listed first is a $\mathrm{Na}^{+}$-dependent system largely specific to glycine. We may define this system from its description by Vidaver ${ }^{10}$ for the pigeon red blood cell. Sarcosine is also reactive with it, but the ordinary amino acids show only small inhibition of glycine uptake. Also listed are two systems with broad reactivities, $A$ and $L$. Almost all ordinary neutral independent System $L$. It is incorrect, however, to speak of alanine as a System $A$ substrate and phenylalanine as a System $L$ substrate; both amino acids are transported by both routes, although to different extents.

Another $\mathrm{Na}^{+}$-dependent system, ASC, is most effective for linear amino acids with 3,4 or 5 atoms in the chain. The presence of a hydroxyl, sulfhydryl or carboxamide group on the sidechain is favorable to their transport. When the hydroxyl group is forced into a particular steric position, high cooperativity with $\mathrm{Na}^{+}$as cosubstrate arises, in a way that permits us to pinpoint the position taken by the sodium ion at the site, adjacent to the amino acid molecule. ${ }^{12}$

Although there are a number of distinctive features, System ASC is most easily differentiated from System $A$ by the high tolerance of the 
latter for an $\mathrm{N}$-methyl group. This tolerance by System $A$ also helps in discriminating transport by Systems $A$ and $L$.

A different transport system serves for basic amino acids, when they are in their cationic forms, and still another one, for acidic amino acids, provided they are in their anionic forms. When the electrical charge on the sidechain is eliminated by a $\mathrm{pH}$ change, these amino acids come to be transported by the neutral systems. Because the Ehrlich ascites tumor cell lacks a system for anionic amino acids, the transport of glutamic acid occurs exclusively by the neutral systems, showing $\mathrm{pH}$ profiles of the shape one would predict from the pK values. More detailed summaries may be found elsewhere, ${ }^{11,12}$ along with the criteria that are considered necessary to the assignments of transport to a given system.

The systematization of amino acid transport has one particular value, that any theory as to how uphill transport is energized must meet the test of corresponding to the systematic classification. In particular a rather large number of artificial amino acids have been found or invented which undergo typical biological transport by a single system, or by more than one system, even though their structures are so unusual that they can undergo little if any metabolic attack and will not undergo, for example, a certain transpeptidation reaction which has long been suspected of a possible role in amino acid transport. The extent to which such proposals fail to correspond to the known properties of the several transport systems should then become a central consideration in testing a new proposal, along with other tests of the specificity of the proposed scheme to amino acid transport.

In the instance of the transpeptidation reaction, the noncorrespondence seems overwhelming. For the present moment, the detailed mechanism by which amino acid transport receives its primary energization in the cell of the higher animal remains controversial and unclear, so that it seems rather early to offer a detailed exposition of mechanism. Some aspects that appear important are: (1) an interchangeability between $\mathrm{Na}^{+}$gradients and amino acid gradients across the plasma membrane $^{11}$ through operation of systems that co- transport the cation and the amino acid; (2) a consequent dependence of the uphill transport of even the neutral amino acids on the transmembrane potential; (3) another mode of energization of the same transport systems, probably even in addition to possible energization through ATP cleavage; ${ }^{13,14}$ (4) an apparent application of the latter mode also to energization of the $\mathrm{Na}^{+}$-independent transport systems.

The transpeptidation cycle illustrates the fondness of enzymologists for schemes that extend to membrane transport the same ideas that apply to enzyme catalysis, i.e., schemes by which the molecule transported necessarily appears in the second phase as a new and different substance-a process called group translocation. A change of the transported solute into something else, however, is not an energetic necessity. Such proposals should meet much more critical consideration than is now often the case outside transport circles. We know that such ions as $\mathrm{Na}^{+}$and $\mathrm{K}^{+}$are not transported in this way; the study of metabolism-resistant amino acid analogs failed to show evidence for an important role for group translocation here also. The discrimination of separate transport systems is obviously of great importance in exploring this matter. A model in which sugars are held to be phosphorylated during transport into yeast has just now been brought into serious question. ${ }^{15}$

It is interesting that the amino acid transport system contributing predominantly to the blood-brain barrier is the $\mathrm{Na}^{+}$-independent one known as System $L .{ }^{16}$ This system is also the main one surviving the maturation of the mammalian red blood cell. ${ }^{17}$ The result is that amino acid molecules with minimal apolarity in their sidechains (e.g., glycine, serine) move into and out of the red blood cell very slowly, while such amino acids as leucine or phenylalanine cross the membrane far more rapidly. A recent observation may mean that glycine transport into and out of the red blood cell in the capillary circulation of the dog may occur much faster than it does when isolated red blood cells are tested. ${ }^{18}$ This result might imply that a defect arising in the transport system on maturation of the reticulocyte could ac- 
tually receive some form of perhaps transient correction when the red blood cell comes into contact with cells of the capillary membrane. It should not be assumed, however, that this possible effect will have importance for the majority of the amino acids, which cross the membrane much faster in vitro than glycine does.

The study of the development of transport during embryonic and early postnatal life, and its response to neoplastic transformation and to derepression also needs to limit its attention to one transport system at a time for clarity to be obtained. This was demonstrated by Guidotti and his associates ${ }^{19}$ in their important studies: The $\mathrm{Na}^{+}$-dependent System $A$ appears in many situations to be the one subject to adaptive control.

1. P. Felig: Amino Acid Metabolism in Man. Ann. Rev. Biochem. 44: 932-955, 1975

2. H.N. Christensen and M.E. Handlogten: Modes of Mediated Exodus of Amino Acids from the Ehrlich Ascites Tumor Cell. J. Biol. Chem. 243: 5428-5438, 1968

3. D.L. Oxender: Stereospecificity of Amino Acid Transport for Ehrlich Tumor Cells. J. Biol. Chem. 240: 2976-2982, 1965

4. H.N. Christensen in Transport Phenomena in the Nervous System, Physiological and Pathological Aspects. G. Levi, L. Battistin and A. Lajtha, Editors, pp. 3-12. Plenum Press, 1973

5. H.N. Christensen: On the Routes of Renal Tubular Resorption of Amino Acid. Proc. 6th Int. Cong. Nephrol., pp. 134-138. Karger, Basel, 1975

6. P. Mitchell: Coupling of Phosphorylation to Electron and Hydrogen Transfer by a ChemiOsmotic Type of Mechanism. Nature 191: 144148, 1961

7. H.N. Christensen: Mass-Action Effects are Indispensable for all the Amino Acids. Nutrition Reviews 34: 30-31, 1976

8. Cyclic Behavior of Amino Acid Transport. Nutrition Reviews 27: 79-81, 1969
9. H.N. Christensen, J.T. Rothwell, R.A. Sears and J.A. Streicher: Association between Rapid Growth and Elevated Cell Concentrations of Amino Acids. II. In Regenerating Liver after Partial Hepatectomy in the Rat. J. Biol. Chem. 175: 101-105, 1948

10. G.A. Vidaver: Transport of Glycine by Pigeon Red Blood Cells. Biochemistry 3:662-667, 1964

11. H.N. Christensen in Current Topics in Membranes and Transport. A. Kleinzeller and F. Bronner, Editors, Vol. 6, pp. 227-258, 1975

12. H.N. Christensen: Hydrogen Dissociation as a Factor in Amino Acid Transport. FEBS Symposium No. 42, Biochemistry of Membrane Transport. G. Semenza and E. Carafoli, Editors, pp. 222-235. Springer, 1977

13. J. Garcia-Sancho, A. Sanchez, M.E. Handlogten and H. N. Christensen: An Unexpected Additional Mode of Energization of Amino Acid Transport into the Ehrlich Cell. Proc. Nat. Acad. Sci. USA 74: 1488-1491, 1977

14. J.A. Schafer and A.E. Williams in Amino Acid Transport and Uric Acid Transport. G. Silbernagel, F. Lang and R. Greger, Editors, pp. 2025. Georg Thieme, Stuttgart, 1977

15. R. Brocklehurst, D. Gardner and A.A. Eddy: The Absorption of Protons with $\alpha$-Methyl Glucoside and $\alpha$-Thioethyl Glucoside by the Yeast N.C.Y.C. 240. Biochem. J. 162: 591-599, 1977

16. L.A. Wade and R. Katzman: Synthetic Amino Acids and the Nature of $L$-DOPA Transport at the Blood-Brain Barrier. J. Neurochem. 25: 837-842, 1975

17. H. N. Christensen and J. A. Antonioli: Differences in Schedules of Regression of Transport Systems during Reticulocyte Maturation. J. Biol. Chem. 244: 1505-1509, 1968

18. D.H. Elwyn, W.J. Launder, H.G. Parikh and E.M. Wise, Jr.: Roles of Plasma and Erythrocytes in Interorgan Transport of Amino Acid in Dogs. Am. J. Physiol. 222: 1333-1342, 1972

19. G.C. Gazzola, R. Franchi-Gazzola, P. Ronchi and G.G. Guidotti: Regulation of Amino Acid Transport in Chick Embryo Heart Cells. III. Formal Identification of the $A$ Mediation as an Adaptive Transport System. Biochem. Biophys. Acta 311: 292-301, 1973 